\title{
First Reported Case of Ceftriaxone-Resistant Typhoid Fever in the Middle-East
}

\author{
Gireesh Kumar Bharathan ${ }^{1 *}$, Binoy Kurian² \\ 'Department of Internal Medicine, Dr. Moopans' Aster Hospital, Doha, Qatar \\ ${ }^{2}$ Department of Microbiology, Dr. Moopen's Aster Hospital, Doha, Qatar
}

Corresponding Author: Gireesh Kumar Bharathan, MD, Department of Internal Medicine, Dr. Moopans' Aster Hospital, Doha, Qatar. Tel: +974-66945086, Email: drgirishvarma@yahoo.co.in

Received June 12, 2020; Accepted September 18, 2020; Online Published November 22, 2020

\begin{abstract}
Introduction: Typhoid fever is a potential febrile illness caused by Salmonella enterica serovar Typhi. It is mainly transmitted through contaminated food and water, and spreads from infected persons and chronic carriers. Humans are the only reservoir host. There were many multi-drug resistant S. Typhi cases reported in many parts of South Asia and Africa. However, since 2016there were reported case series of extended drug resistant $S$. Typhi from Pakistan (resistant to chloramphenicol, ampicillin, trimethoprim-sulfamethoxazole, fluoroquinolones and third generation cephalosporines). Genomic sequencing analysis of this organism was of haplotype H58 transmitted by a plasmid.

Case Presentation: This is the first reported case of ceftriaxone-resistant typhoid fever in the Middle-East, who travelled from Pakistan to Qatar in September 2019. He recovered completely with injection meropenem and oral azithromycin.

Conclusion: A detailed travel history and early diagnosis and treatment with appropriate drugs are inevitable in the management of such cases. Safe drinking water, improved sanitation and effective public health infrastructure will play a key role in the control of the disease. Keywords: Salmonella Typhi, Antimicrobial Resistance, Enteric Fever
\end{abstract}

Citation: Bharathan GK, Kurian B. First reported case of ceftriaxone-resistant typhoid fever in the Middle-East. Int J Travel Med Glob Health 2021;9(1):39-41. doi:10.34172/ijtmgh.2021.07.

\section{Introduction}

Typhoid fever is a potential febrile illness, caused by Salmonella enterica serovar Typhi, which is most commonly seen in South-East Asia and Africa. Some cases can have a severe debilitating course, leading to life-threatening conditions. Its infection is restricted only to a human host with an incubation period of 6-30 days. This bacterium is transmitted through contaminated water and food, and tends to spread in the areas of poor sanitation. The escalating antimicrobial resistance in $S$. Typhi in the last few decades is a global concern in the management of typhoid. ${ }^{1}$

With reference to the reports from United States Communicable Disease Centre, about 26 million cases of typhoid fever occur worldwide each year, causing 2,15,000 deaths. ${ }^{2}$ Surveillance for Enteric Fever in Asia Project (SEAP) has established drug resistance to multiple antimicrobials including fluoroquinolones, chloramphenicol, ampicillin, and trimethoprim-sulfamethoxazole. ${ }^{3}$ During 2016-2018, several cases of cephalosporin resistant typhoid cases were reported in Pakistan. ${ }^{4}$ Later, there were many reported cases of international transmission of Extensively Drug Resistant (XDR) S. Typhi strain from the United States, United Kingdom and Australia. In the United States itself, 5 cases of XDR typhoid were reported in 2016-2018, who travelled to or from Pakistan during that period ${ }^{5}$. There has been no reported cases of ceftriaxone-resistant $S$. Typhi in the MiddleEast. This article describes a case of $S$. Typhi infection, who travelled from Pakistan.

\section{Case Presentation}

A 44-year-old man returned from Lahore, Pakistan, with a history of high-grade fever for 10 days with severe headache, fatigue and loss of appetite. He was started on cefixime (400 mg) -BID by a local doctor. He also gave a history of food intake from the outside of home in Pakistan and there was no previous history of vaccination for typhoid.

On examination, he was febrile, toxic and dehydrated with low blood pressure of 90/60 mm Hg. After taking blood samples for culture and other preliminary investigation, he was started on injection. Ceftriaxone and azithromycin along

Copyright $(\odot) 2021$ The Author(s). This is an open-access article distributed under the terms of the Creative Commons Attribution License (http:// creativecommons.org/licenses/by/4.0), which permits unrestricted use, distribution, and reproduction in any medium, provided the original work is properly cited. 
with other supportive medications. From day 1 of admission, high-grade fever with shivering and severe headache was recorded every 6 hours which continued in spite of taking antipyretic medication. His serum C-reactive protein and liver enzymes were elevated. Radiological imaging of chest and abdomen were unremarkable (Table 1).

After 24 hours of admission, his blood culture which was performed by BD Phoenix M50 system showed the growth of gram-negative organism. Regarding antimicrobial stewardship, he was switched over to IV meropenam $1 \mathrm{gm}$ thrice daily. Later, $S$. Typhi was isolated from the blood, which was resistant to all cephalosporins, so he was continued on injection (Inj) meropenem. After 48 hours of Inj. meropenem, the number of spikes of fever reduced and his blood pressure improved. On day 7 of Inj. meropenem, defervescence was started, and he became afebrile and was discharged from hospital on oral azithromycin. After 5 days of discharge, he was reviewed in the outpatient department and his clinical condition was shown to have improved significantly except for mild fatigue. His serum C-reactive protein level reduced to near normal (Table 2).

\section{Discussion}

To the best of our knowledge, this is one of the first reported cases of ceftriaxone-resistant S. Typhi in the Middle East. Typhoid fever is caused by gram-negative organism, $S$. enterica serovar Typhi. It spreads through the food and water contaminated with human wastes. In the absence of sensitive antibiotics, typhoid fever will have a prolonged and debilitating course which may lead to the development of severe complications and death. ${ }^{6}$

Salmonella Typhi had shown anti-microbial resistance to multiple drugs since 1970s relentlessly till the early 1990s. Fluroquinolones then emerged as the treatment of choice in 1990s. ${ }^{7}$ Wide spread irrational use of these antibiotics led to reduced susceptibility to $S$. Typhi. Later, the outbreak of fluroquinolone resistant typhoid cases was reported in different parts of the world. Thus Inj. Ceftriaxone and oral Cefixime became the mainstay of the management of typhoid. ${ }^{8}$

The outbreak of typhoid occurred in Pakistan during 2016-2018, isolated strains having haplotype-58 which were resistant to first- and second-line drugs and third generation cephalosporins (XDR). The XDR S. Typhi strain developed the resistance via a plasmid. In our case, $S$. Typhi showed resistance to cephalosporins but was susceptible to carbapenems, piperacillin-tazobactam and tigecycline. However, the sensitivity of azithromycin and other macrolides were not checked. Also, the genomic sequencing of $S$. Typhi could not be performed in our study.

Isolation of $S$. Typhi in blood and bone marrow remains the definitive diagnostic test for Typhoid fever, while the serological test is discouraged due to the lack of accuracy. ${ }^{9}$ The delay in diagnosis and management of sensitive antibiotics can lead to many complications. The XDR salmonella are more prone to develop complications like massive gastrointestinal bleeding, thickening of ileo-caecal region, large soft tissue abscess and significant mesenteric lymphadenopathy. ${ }^{10}$

In many developing countries, typhoid fever is a public health problem. ${ }^{11}$ Unsafe water, poor sanitation, inadequate public health infrastructure and lack of awareness among public make the situation more abysmal. Safe drinking water, improved sanitation and public health infrastructure will play the key role in the control of the disease from the source. Due to an increase in immigration, there is a high probability of the spread of the disease world-wide. Sporadic cases are already reported in developed countries.

Vaccination is an important and effective tool in preventing this disease, especially when travelling to the typhoid endemic areas. The World Health Organization recommends two types of safe vaccines for typhoid-oral and injectable. ${ }^{12}$ Oral vaccine is approved for people of 6 years and older. It should be taken at least 1 week prior to travel. The injectable vaccine Typhi IV is approved for 2-year-old individuals and older. Travellers should receive injections at least 2 weeks before departure.

Effective health education among public and health care workers is the cornerstone for the control of spread of the drug resistant typhoid. Frequent inspection in restaurants, health checkup among food handlers and enactment of the law are also very helpful in preventing food-borne diseases like typhoid. ${ }^{13}$

In the last seven decades, the $S$. Typhi has shown resistance to multiple antibiotics by different mechanism. It also necessitated the judicial use of antibiotics by health care professionals under antimicrobial stewardship to prevent the development of its resistance and thus the global threat by the micro-organism to the human society at large. ${ }^{1}$

Table 1. Lab Reports

\begin{tabular}{|c|c|c|c|c|c|c|}
\hline TEST & Day1 & Day3 & Day7 & Day 11 & Day16 & Unit \\
\hline Hemoglobin & 13.66 & & & & 13.98 & $\mathrm{~g} / \mathrm{dL}$ \\
\hline Platelet count & 278.2 & & & & 337.8 & $10^{\wedge} 3 / \mathrm{uL}$ \\
\hline Neutrophils & 59 & & & & 48 & $\%$ \\
\hline Lymphocytes & 31 & & & & 42 & $\%$ \\
\hline Monocytes & 08 & & & & 08 & $\%$ \\
\hline Eosinophils & 02 & & & & 02 & $\%$ \\
\hline Basophils & 00 & & & & 00 & $\%$ \\
\hline Alanine aminotransferase (ALT), serum & 109 & 112 & 176 & & 138.5 & $\mathrm{mg} / \mathrm{dL}$ \\
\hline C-reactive protein serum (quantitative) & 53.8 & & 40.95 & 21.87 & 8.9 & $\mathrm{mg} / \mathrm{dL}$ \\
\hline Creatinine, serum & 0.7 & & & & 0.86 & $\mathrm{mg} / \mathrm{dL}$ \\
\hline
\end{tabular}


Table 2. Susceptibility Report

\begin{tabular}{lcc}
\hline Antimicrobial & MIC or Concentration & Final SIR \\
\hline Amikacin & $\leq 8$ & $\mathrm{R}$ \\
Cefepime & $>16$ & $\mathrm{R}$ \\
Ceftazidime & $>16$ & $\mathrm{R}$ \\
Ceftriaxone & $>32$ & $\mathrm{R}$ \\
Cefuroxime & $>16$ & $\mathrm{R}$ \\
Ciprofloxacin & $\leq 0.5$ & $\mathrm{I}$ \\
Imipenem & $\leq 1$ & $\mathrm{~S}$ \\
Meropenem & $\leq 1$ & $\mathrm{~S}$ \\
Trimethoprim-sulphamethoxazole & $<4 / 76$ & $\mathrm{R}$ \\
\hline
\end{tabular}

Abbreviations: MIC, minimum inhibitory concentration; SIR, sensitive, intermediate, resistant; S, Sensitive; I, Intermediate; R, Resistant.

\section{Conclusion}

The ceftriaxone-resistant typhoid fever is a global concern and a public health problem. The lack of early diagnostic tools and limited options in the pharmacological armamentarium are practical hurdles in the medical management of typhoid fever. It highlights the need for more research in this field. Effective vaccination, good sanitation and healthy lifestyles can prevent the spread of the disease to a great extent.

\section{Authors' Contributions}

GKB and BK wrote the first draft of the manuscript. GKB performed the diagnosis and patients' management and was in charge of follow-up. BK performed laboratory analysis and provided scientific contribution. BK also critically revised the paper. Both authors have read and agreed to the published version of the manuscripts.

\section{Conflict of Interest Disclosures}

None declared.

\section{Ethical Approval}

This case report was approved by the Ethics committee of Dr. Moopens Aster Hospital, Qatar. A written informed consent for the evaluation was obtained from the patient. The Ethics Committee of DM Aster Hospital, Qatar, has also approved the publication of the case report and the written consent form from patient.

\section{Funding/Support}

None.

\section{References}

1. Tangcharoensathien V, Sattayawutthipong W, Kanjanapimai S, Kanpravidth W, Brown R, Sommanustweechai A. Antimicrobial resistance: from global agenda to national strategic plan, Thailand. Bull World Health Organ. 2017;95(8):599-603. doi:10.2471/ blt.16.179648.

2. Appiah GD, Hughes MJ, Chatham-Stephens K. Typhoid \& Paratyphoid Fever. Centers for Disease Control and Prevention (CDC); 2020. https://wwwnc.cdc.gov/travel/yellowbook/2020/ travel-related-infectious-diseases/typhoid-and-paratyphoid-fever. Accessed June 5, 2020.

3. Barkume C, Date K, Saha SK, et al. Phase I of the Surveillance for Enteric Fever in Asia Project (SEAP): an overview and lessons learned. J Infect Dis. 2018;218(Suppl_4):S188-S194. doi:10.1093/ infdis/jiy522.

4. World Health Organization (WHO). Emergencies Preparedeness Response - Typhoid Fever-Islamic Republic of Pakistan. WHO; 2018.

5. Chatham-Stephens K, Medalla F, Hughes M, et al. Emergence of extensively drug-resistant Salmonella Typhi infections among travelers to or from Pakistan - United States, 2016-2018. MMWR Morb Mortal Wkly Rep. 2019;68(1):11-13. doi:10.15585/mmwr. mm6801a3.

6. World Health Organization (WHO). Typhoid. https://www.who. int/news-room/fact-sheets/detail/typhoid. Published January 31, 2018. Accessed June 2020 5,

7. Jesudasan M, John TJ, Gupta BL, Bhujwala RA, Shriniwas. Multiresistant Salmonella typhi in India. The Lancet. 1990; 336(8709):252. doi:10.1016/0140-6736(90)91783-7.

8. Browne AJ, Kashef Hamadani BH, Kumaran EAP, et al. Drugresistant enteric fever worldwide, 1990 to 2018: a systematic review and meta-analysis. BMC Med. 2020;18(1):1. doi:10.1186/ s12916-019-1443-1.

9. Crump JA, Sjölund-Karlsson M, Gordon MA, Parry CM. Epidemiology, clinical presentation, laboratory diagnosis, antimicrobial resistance, and antimicrobial management of invasive Salmonella infections. Clin Microbiol Rev. 2015;28(4):901-937. doi:10.1128/cmr.00002-15.

10. Bhatti JM, Memon Y, Sarfaraz S, Salahuddin N. An unusual case of extensively drug resistant typhoid fever. Cureus. 2019;11(5):e4664. doi:10.7759/cureus.4664.

11. Divyashree S, Nabarro LE, Veeraraghavan B, Rupali P. Enteric fever in India: current scenario and future directions. Trop Med Int Health. 2016;21(10):1255-1262. doi:10.1111/tmi.12762.

12. World Health Organization (WHO). Immunization Vaccines and Biologicals. https://www.who.int/ith/vaccines/typhoidfever/en. Published December 9, 2019. Accessed January 1, 2020.

13. World Health Organization (WHO). Foodborne Disease Outbreaks: Guidelines for Investigation and Control. WHO; 2008. https://www.who.int/foodsafety/publications/foodborne_disease/ outbreak_guidelines.pdf. 